\title{
Prevalence and Diagnosis of Sesame Allergy in Children with IgE-Mediated Food Allergy
}

\author{
Kristin Sokol, MD ${ }^{\mathrm{a}}$, Marjohn Rasooly, MSN ${ }^{\mathrm{a}}$, Caeden Dempsey, RN, MPH ${ }^{\mathrm{a}}$, Sheryce \\ Lassiter, MSN ${ }^{b}$, Wenjuan Gu, PhD $^{b}$, Keith Lumbard, $\mathbf{M S}^{b}$, Pamela A Frischmeyer-Guerrerio, \\ MD PhDa \\ aLaboratory of Allergic Diseases, Food Allergy Research Unit, NIAID, NIH, Bethesda, MD \\ ${ }^{b}$ Clinical Research Directorate, Frederick National Laboratory for Cancer Research sponsored by \\ the National Cancer Institute
}

\section{Short Summary}

iii.

Sesame allergy is estimated to affect $17 \%$ of children with IgE-mediated food allergy, and often co-occurs with peanut/tree nut sensitization and allergy. Sesame-specific IgE levels may have utility in the clinical management of these patients.

\section{Keywords}

Sesame; sesame allergy; food allergy; peanut allergy; tree nut allergy; diagnosis; prevalence; sensitization

To the Editor:

Sesame has emerged as an important food allergen around the world, including the United States (U.S.) where it is the ninth most common childhood food allergy with a population prevalence of approximately $0.1-0.2 \% .{ }^{1}$ Sesame is the most common seed to cause hypersensitivity reactions, likely due to its widespread use in the food, pharmaceutical, and cosmetic industries. The U.S. Food and Drug Administration (FDA) is currently considering a petition to include sesame in the list of allergens that must be disclosed on food labels as is currently mandated in Europe, Australia, and Canada. Only an estimated $20-30 \%$ of patients outgrow their sesame allergy, and severe anaphylactic reactions are common, underscoring the need to optimize recognition and diagnosis of this allergy. ${ }^{2}$

The diagnosis of sesame allergy is challenging as there are no identified sesame specific IgE (sIgE) or skin prick test (SPT) thresholds that predict clinical reactivity. Recently, specific component testing against a recombinant Sesi 1 allergen has been reported, with serum reactivity to Sesi 1 yielding a specificity and sensitivity of $86.1 \%$ and 85.7 respectively. ${ }^{3}$

Corresponding Author: Pamela A. Guerrerio, MD PhD, Building 10, Room 11N240B, MSC 1889, 10 Center Drive, Bethesda, MD 20892, Phone: (301) 402-9782, Fax: (301) 480-4507, pamela.guerrerio@nih.gov.

i. Conflicts of Interest

None 
However, component testing for sesame is not currently available clinically. Many prior studies evaluating the utility of SPT and sesame sIgE testing as a diagnostic tool included children with a suspicion for sesame allergy. ${ }^{4,5}$ The goal of this study was to examine the prevalence and manifestations of sesame allergy in a population of U.S. children with IgEmediated food allergy and to determine the utility of sIgE testing in predicting sesame allergy in this cohort.

One hundred nineteen consecutive subjects (median age 9.0 years; $61.3 \%$ male) with IgEmediated food allergy enrolled on a Natural History of Food Allergy protocol at the National Institutes of Health (NIH) were assessed for sensitization and clinical reactivity to sesame (Table 1). Subjects with a history of an immediate hypersensitivity reaction to at least one food along with positive IgE testing to that food were eligible for enrollment. No eligible subjects declined participation. All subjects were offered an oral food challenge (OFC) to sesame unless they had a recent convincing history of a hypersensitivity reaction (within 3 years) to sesame or if they were known to tolerate concentrated sesame (tahini, hummus) in their diet. Sesame sensitization was defined as a sesame $\operatorname{sIgE}>0.35 \mathrm{kU}_{\mathrm{A}} / \mathrm{L}$, sesame allergy as a recent immediate hypersensitivity reaction following exposure to sesame at home or via clinic food challenge (tahini), and sesame tolerance as the ability to ingest concentrated sesame (tahini, hummus) with no symptoms. For clinic OFCs ( $\mathrm{n}=11)$, increasing doses of tahini, starting with $0.045 \mathrm{~g} \mathrm{(1 \% )}$ of sesame protein, were administered every 15-30 minutes up to a cumulative dose of at least $4.5 \mathrm{~g}$. An OFC was considered positive if the subject experienced objective symptoms (based on PRACTALL guidelines) ${ }^{6}$, and negative if symptoms were absent after ingesting all doses. Food-specific and total IgE were measured by ImmunoCAP (Thermo Fisher, Uppsala, Sweden). The study was approved by the NIH IRB and informed consent/assent was obtained for all subjects. Comparisons between groups were made with Wilcoxon rank sum tests, Chi-squared tests, or Fishers Exact tests. Logistic regression was used to estimate predictors of allergy. P-values <.05 were considered statistically significant.

Of the 119 patients, $108(91 \%)$ were sensitized to sesame, 15 (12.6\%) were confirmed sesame allergic and 73 (61.3\%) were sesame tolerant (Table 1). Although all subjects were offered an OFC to sesame regardless of their sesame sIgE levels, sesame allergy status could not be determined for 31 (26.1\%) subjects, most of whom reported no known exposure to sesame and were avoiding it as a result of previous positive sIgE testing. There were no statistically significant differences in age, gender, ethnicity, allergic comorbidities, total reported food allergies (excluding sesame), or total IgE levels between those allergic vs. tolerant to sesame (Table 1). Among the 88 subjects with confirmed allergic status, the prevalence of sesame allergy was $17.0 \%$. Compared to those who were tolerant, sesame allergic subjects had higher sesame sIgE levels (median 31.2 vs. $2.2 \mathrm{kU}_{\mathrm{A}} / \mathrm{L}$ ) and sesame sIgE/total IgE ratio (median 0.029 vs. 0.002) (Table 1; Fig. 1A, B). An ROC analysis (Fig. 1C, D) and a univariate logistic model (Fig. 1E, F) were performed on the 88 subjects with confirmed sesame allergic status. The sesame specific IgE threshold that maximized the sum of estimated sensitivity and specificity was $11.6 \mathrm{kU}_{\mathrm{A}} / \mathrm{L}(4.9,19.7)$ with a sensitivity of 0.93 and a specificity of 0.85 (Fig. 1C). Using logistic regression, another threshold was estimated as the value associated with $50 \%$ probability of tolerating sesame: $29.4 \mathrm{kUA} / \mathrm{L}$ $(17.4,61.2)$ (Fig. 1E). Dividing sesame IgE by total serum IgE did not appear to improve the 
ability of sesame sIgE to predict allergy in this cohort (Fig. 1D, F). However, logistic regression analysis found that a model with the two variables, total IgE and sesame sIgE, provided statistically significant better prediction of sesame allergy than a model with sesame sIgE alone.

Sesame oil and intact seeds are sometimes considered less allergenic than sesame paste since highly processed oils may contain less intact protein and seeds can be passed without significant digestion; however, anaphylaxis to these forms of sesame has been reported. ${ }^{7,8}$ Of the 15 sesame allergic subjects in this study, 4 could tolerate sesame oil and 2 could tolerate sesame seeds without any symptoms (Table E1). On the other hand, 3 subjects reported reactions to sesame oil and 7 to sesame seeds. The remaining subjects had never tried sesame oil or seeds since their initial allergic reaction was after ingestion of concentrated sesame and they were advised to subsequently avoid all sesame products (Table E1). Many of the sesame allergic patients were able to tolerate other seeds, and in fact, none reported an allergic reaction to other seeds although nearly half of the subjects had not ingested other seeds (Table E1).

Both serologic and clinical cross-reactivity has been observed between sesame and peanut and tree nuts. ${ }^{2}$ In this cohort, $93.3 \%$ of subjects with confirmed sesame allergy were sensitized to peanut and $100 \%$ to at least one tree nut, compared to $84.9 \%$ and $90.4 \%$, respectively, in those with known tolerance to sesame. Of the 15 patients allergic to sesame, $6(40.0 \%)$ had a history of an acute hypersensitivity reaction to peanut and $6(40.0 \%)$ to at least one tree nut, compared to $32(43.8 \%)$ and $14(19.2 \%)$ in the tolerant group. In general, subjects with sesame allergy had higher rates of allergy to individual tree nuts, although this did not always reach statistical significance (Table 1). Of note, a large group of subjects with confirmed sesame allergy and tolerance had never ingested peanut $(n=7 ; 46.7 \% ; n=26$; $35.6 \%$, respectively) or any tree nuts $(n=8 ; 53.3 \% ; n=45 ; 61.6 \%$, respectively). Peanut and tree nut sIgE levels were generally higher in allergic than tolerant subjects (Table 1).

The diagnosis of sesame allergy remains a clinically challenging problem. Our results suggest that sesame IgE levels may have diagnostic utility. A recent study also found sesame IgE levels to be significantly higher in children allergic versus tolerant to sesame, although Ses i 1-IgE levels were most effective at predicting the result of an OFC for sesame while sesame SPT was ineffective, in contrast to other studies ${ }^{9,10}$ that have found SPT to be predictive. Sesame component IgE and skin prick testing were not performed in this study, and therefore we cannot comment on the relative utility of these different diagnostic tools.

One major limitation of this study is that allergic status to sesame could not be determined for $31(26.1 \%)$ patients, as they had never consumed concentrated sesame. These subjects either declined a sesame OFC ( $n=24$; reasons included anxiety, distance from our institution, or no interest in eating sesame-containing products), or had medical conditions such as active eosinophilic esophagitis that contraindicated an OFC $(n=7)$. Over half of these 31 subjects who had never ingested concentrated sesame were tolerating sesame seeds and/or oil without a subsequent hypersensitivity reaction. The sesame IgE level of those with unknown sesame allergic status was intermediate $\left(8.92 \mathrm{kU}_{\mathrm{A}} / \mathrm{L}\right)$ between those with confirmed sesame allergy and tolerance, and thus the true prevalence of sesame allergy in 
our cohort is likely to be higher than $12.6 \%$ (Fig. E1, Table E1). Other clinical and serologic features of those with unknown status are listed in Table E1. Additionally, subjects were required to have IgE-mediated food allergy to enroll in our study, and therefore our findings may not be applicable to the general population. Our cohort may also represent a more severely affected group of children with food allergy as the majority were allergic to more than one food. Sesame component testing was not performed and therefore we are not able to distinguish whether the high rate of peanut and tree nut sensitization and allergy in this cohort represents sensitization to cross-reactive epitopes or co-reactivity. For example, the major peanut allergens 7S globulin (Ara h1) and 11S globulin (Ara h3) are highly homologous (including IgE-binding epitopes) to the corresponding proteins in sesame. ${ }^{11} \mathrm{~A}$ major strength of our study is that consecutive patients with IgE-mediated food allergy were enrolled as opposed to only those with suspected sesame allergy or known sensitization, which distinguishes our approach from many prior studies.

In summary, sesame allergy is common among children with IgE-mediated food allergy, and often co-occurs with peanut and tree nut sensitization and allergy. A subset of sesame allergic children are able to tolerate sesame seeds and oil, although these products can cause severe reactions in some patients. Sesame-specific IgE levels were identified that may be helpful in deciding when to consider oral food challenges.

\section{Supplementary Material}

Refer to Web version on PubMed Central for supplementary material.

\section{Acknowledgements}

We acknowledge Erica Brittain PhD for her advice with the statistical analysis and helpful reading of the manuscript.

ii. Funding and Disclaimers

This research was supported by the Intramural Research Program of the National Institute of Allergy and Infectious Diseases, NIH. This project was funded in part with federal funds from the National Cancer Institute, National Institutes of Health under Contract No. HHSN26120080000IE (to W.G, S.L., and K.L.). The content of this publication does not necessarily reflect the views or policies of the Department of Health and Human Services, nor does mention of trade names, commercial products, or organizations imply endorsement by the U.S. Government.

\section{Abbreviations}

$\begin{array}{ll}\text { US } & \text { United States } \\ \text { FDA } & \text { Food and Drug Administration } \\ \text { SIgE } & \text { specific IgE } \\ \text { SPT } & \text { skin prick test } \\ \text { OFC } & \text { oral food challenge } \\ \text { NIH } & \text { National Institutes of Health } \\ \text { ROC } & \text { Receiver Operating Characteristic }\end{array}$




\section{References}

1. Gangur V, Kelly C, Navuluri L. Sesame allergy: a growing food allergy of global proportions? Ann Allergy Asthma Immunol 2005; 95:4-11; quiz -3, 44. [PubMed: 16095135]

2. Tuano KT, Dillard KH, Guffey D, Davis CM. Development of sesame tolerance and cosensitization of sesame allergy with peanut and tree nut allergy in children. Ann Allergy Asthma Immunol 2016; 117:708-10. [PubMed: 28073703]

3. Maruyama N, Nakagawa T, Ito K, Cabanos C, Borres MP, Moverare R, et al. Measurement of specific IgE antibodies to Ses i 1 improves the diagnosis of sesame allergy. Clin Exp Allergy 2016; 46:163-71. [PubMed: 26310924]

4. Permaul P, Stutius LM, Sheehan WJ, Rangsithienchai P, Walter JE, Twarog FJ, et al. Sesame Allergy: Role of Specific IgE and Skin Prick Testing in Predicting Food Challenge Results. J Allergy Clin Immunol 2009; 123:S24. [PubMed: 21743756]

5. Zavalkoff S, Kagan R, Joseph L, St-Pierre Y, Clarke A. The value of sesame-specific IgE levels in predicting sesame allergy. J Allergy Clin Immunol 2008; 121:1508-10. [PubMed: 18539199]

6. Sampson HA, Gerth van Wijk R, Bindslev-Jensen C, Sicherer S, Teuber SS, Burks AW, et al. Standardizing double-blind, placebo-controlled oral food challenges: American Academy of Allergy, Asthma \& Immunology-European Academy of Allergy and Clinical Immunology PRACTALL consensus report. J Allergy Clin Immunol 2012; 130:1260-74. [PubMed: 23195525]

7. Asero R, Mistrello G, Roncarolo D, Antoniotti PL, Falagiani P. A case of sesame seed-induced anaphylaxis. Allergy 1999;54(5):526-7. [PubMed: 10380788]

8. Sesame seed and sesame seed oil contain masked allergens of growing importance. Allergy. 1996:51;952-7. [PubMed: 9020427]

9. Yanagida N, Eiri Y, Takeishi D, Sato S, Maruyama N, Takahashi K, et al. Ses i 1-specific IgE and sesame oral food challenge results. J Allergy Clin Immunol Pract. 2019;7(6):2084-6. [PubMed: 30836231]

10. Peters RL, Allen KJ, Dharmage SC, Tang MLK, Koplin JJ, Ponsonby A, et al. Skin prick test responses and allergen-specific IGE levels as predictors of peanut, egg, and sesame allergy in infants. J Allergy Clin Immunol. 2013;132(4):874-80. [PubMed: 23891354]

11. Beyer K, Grishina Bardina L, Sampson HA. Identidication of 2 new sesame seef allergens: Ses i 6 and Ses i 7. J Allergy Clin Immunol 2007;119:1554-6. [PubMed: 17556061] 

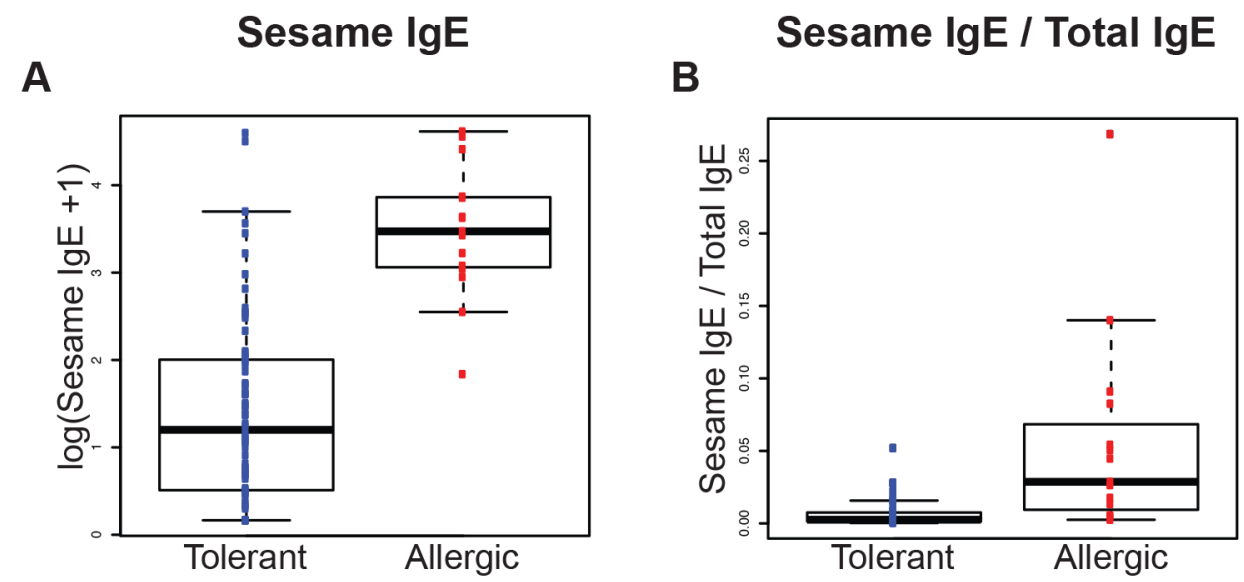

C

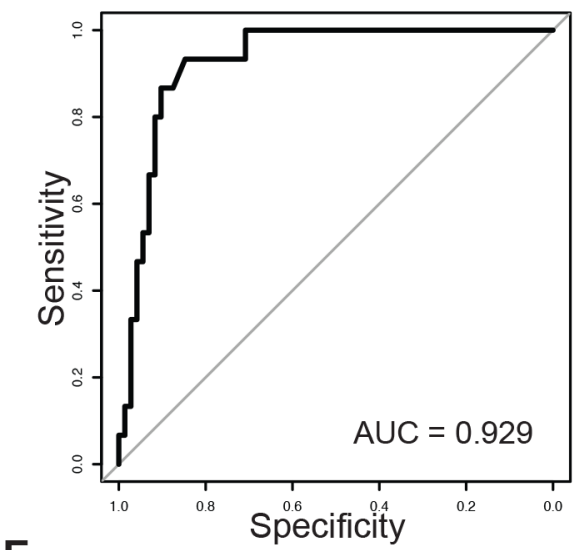

D

$\mathbf{E}$

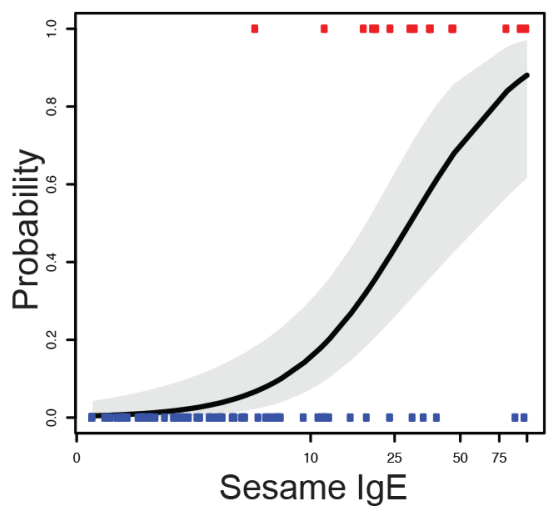

$\mathbf{F}$
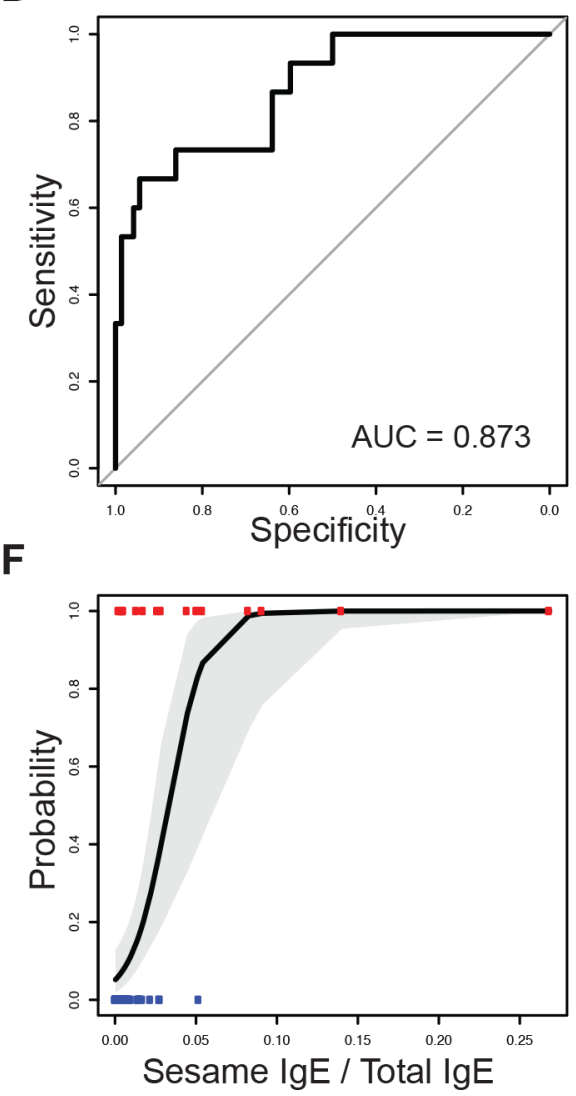

Fig 1.

Sesame-specific IgE and ratio of sesame-specific/total $\operatorname{IgE}$ in subjects allergic or tolerant to sesame. Box plots (A and $\mathbf{B})$ and ROC curves $(\mathbf{C}, \mathbf{D})$ depicting $\log ($ sesame $\operatorname{sIgE}+1)(\mathbf{A}, \mathbf{C})$ and ratio of sesame $\operatorname{IgE} /$ total $\operatorname{IgE}(\mathbf{B}, \mathbf{D})$ in subjects allergic (red dots) or tolerant (blue dots) to sesame; logistic regression analysis $(\mathbf{E}, \mathbf{F})$ for sesame IgE and sesame IgE/total. 
Table 1.

Characteristics of study participants (number, \%)

\begin{tabular}{|c|c|c|c|c|c|}
\hline & & Overall $(n=119)$ & $\begin{array}{c}\text { Sesame Allergic } \\
(\mathrm{n}=15)\end{array}$ & $\begin{array}{c}\text { Sesame Tolerant } \\
(\mathrm{n}=\mathbf{7 3})\end{array}$ & p-value ${ }^{\dagger}$ \\
\hline \multicolumn{2}{|c|}{ Age (years) ${ }^{*}$} & $9.0(5.0,14.0)$ & $9.0(4.0,14.0)$ & $9.0(5.0,14.0)$ & 0.96 \\
\hline \multirow[t]{5}{*}{ Ethnicity: } & Asian & $16(13.4 \%)$ & $2(13.3 \%)$ & $10(13.7 \%)$ & \multirow{5}{*}{0.37} \\
\hline & Black & $15(12.6 \%)$ & $4(26.7 \%)$ & $7(9.6 \%)$ & \\
\hline & Hispanic & $4(3.4 \%)$ & 0 & $3(4.1 \%)$ & \\
\hline & Mixed & $10(8.4 \%)$ & $2(13.3 \%)$ & $7(9.6 \%)$ & \\
\hline & White & $74(62.2 \%)$ & $7(46.7 \%)$ & $46(63.0 \%)$ & \\
\hline \multirow[t]{2}{*}{ Gender: } & Female & $46(38.7 \%)$ & $4(26.7 \%)$ & $33(45.2 \%)$ & \multirow{2}{*}{0.30} \\
\hline & Male & $73(61.3 \%)$ & $11(73.3 \%)$ & $40(54.8 \%)$ & \\
\hline \multicolumn{2}{|l|}{ Asthma } & $71(59.7 \%)$ & $7(46.7 \%)$ & $44(60.3 \%)$ & 0.49 \\
\hline \multicolumn{2}{|c|}{ Atopic dermatitis } & $97(81.5 \%)$ & $13(86.7 \%)$ & $55(75.3 \%)$ & 0.54 \\
\hline \multicolumn{2}{|c|}{ Allergic rhinitis } & $101(84.9 \%)$ & $12(80.0 \%)$ & $60(82.2 \%)$ & 1.00 \\
\hline \multicolumn{2}{|l|}{ EGID } & $17(14.3 \%)$ & $3(20.0 \%)$ & $7(9.6 \%)$ & 0.48 \\
\hline \multicolumn{2}{|c|}{ Total number of reported non-sesame food allergies } & $4.0(2.0,7.0)$ & $5.0(3.5,7.0)$ & $3.0(2.0,5.0)$ & 0.07 \\
\hline \multicolumn{2}{|c|}{ Total IgE $(\mathrm{kU} / \mathrm{L})^{*}$} & $\begin{array}{l}1067.0(350.0 \\
2306.0)\end{array}$ & $975.0(463.0,1754.0)$ & $542.0(306.0,1854.0)$ & 0.28 \\
\hline \multicolumn{2}{|c|}{ Sesame $\operatorname{IgE}\left(\mathrm{kU}_{\mathrm{A}} / \mathrm{L}\right)^{*}$} & $4.5(1.0,20.5)$ & $31.2(20.0,46.3)$ & $2.2(0.6,6.0)$ & $<0.0001$ \\
\hline \multicolumn{2}{|c|}{ Sesame sensitized ${ }^{\mathcal{S}}$} & $108(90.8 \%)$ & $15(100 \%)$ & $62(84.9 \%)$ & 0.24 \\
\hline \multicolumn{2}{|c|}{ Sesame sIgE/Total IgE ratio* } & $0.004(0.002,0.011)$ & $0.029(0.005,0.054)$ & $0.002(0.001,0.007)$ & $<0.0001$ \\
\hline \multicolumn{2}{|c|}{ Peanut sensitized ${ }^{\mathcal{S}}$} & $106(89.1 \%)$ & $14(93.3 \%)$ & $62(84.9 \%)$ & 0.65 \\
\hline \multicolumn{2}{|c|}{ Peanut allergic ${ }^{*}$} & $48(40.3 \%)$ & $6(40.0 \%)$ & $32(43.8 \%)$ & 1.00 \\
\hline \multicolumn{2}{|c|}{ Peanut $\operatorname{IgE}\left(\mathrm{kU}_{\mathrm{A}} / \mathrm{L}\right)^{*}$} & $29(3.0,100)$ & $53.5(8.2,78.1)$ & $13.5(1.5,100)$ & 0.27 \\
\hline \multicolumn{2}{|c|}{ Any TN sensitized ${ }^{\mathcal{S}}$} & $111(93.3 \%)$ & $15(100 \%)$ & $66(90.4 \%)$ & 0.47 \\
\hline \multicolumn{2}{|c|}{ Any $\mathrm{TN}$ allergic } & $24(20.2 \%)$ & $6(40.0 \%)$ & $14(19.2 \%)$ & 0.16 \\
\hline \multicolumn{2}{|c|}{ Almond sensitized $^{\mathcal{S}}$} & $26(23 \%)$ & $15(100 \%)$ & $24(32.8 \%)$ & $<0.0001$ \\
\hline \multicolumn{2}{|c|}{ Almond allergic ${ }^{*}$} & $7(5.9 \%)$ & $2(13.3 \%)$ & $2(2.7 \%)$ & 0.27 \\
\hline \multicolumn{2}{|c|}{ Almond $\operatorname{IgE}\left(\mathrm{kU}_{\mathrm{A}} / \mathrm{L}\right)^{*}$} & $2.1(0.5,7.8)$ & $4.4(2.0,7.2)$ & $1.1(0.2,4.5)$ & 0.02 \\
\hline \multicolumn{2}{|c|}{ Brazil nut sensitized $\xi$} & $72(60.5 \%)$ & $12(80.0 \%)$ & $37(50.7 \%)$ & 0.07 \\
\hline \multicolumn{2}{|c|}{ Brazil nut allergic ${ }^{*}$} & 0 & 0 & 0 & $1.00^{\&}$ \\
\hline \multicolumn{2}{|c|}{ Brazil nut $\operatorname{IgE}\left(\mathrm{kU}_{\mathrm{A}} / \mathrm{L}\right)^{*}$} & $1.3(0.2,7.8)$ & $1.7(0.7,4.0)$ & $0.7(0.2,3.8)$ & 0.36 \\
\hline \multicolumn{2}{|c|}{ Cashew sensitized $^{\mathcal{\xi}}$} & $94(79.0 \%)$ & $14(93.3 \%)$ & $52(71.2 \%)$ & 0.14 \\
\hline \multicolumn{2}{|c|}{ Cashew allergic ${ }^{t^{t}}$} & $13(10.9 \%)$ & $2(13.3 \%)$ & $9(12.3 \%)$ & 1.00 \\
\hline \multicolumn{2}{|c|}{ Cashew $\operatorname{IgE}\left(\mathrm{kU}_{\mathrm{A}} / \mathrm{L}\right)^{*}$} & $5.0(0.7,24.0)$ & $5.0(0.9,19.9)$ & $2.8(0.2,16.4)$ & 0.57 \\
\hline
\end{tabular}

Pediatr Allergy Immunol. Author manuscript; available in PMC 2021 February 01. 


\begin{tabular}{|c|c|c|c|c|}
\hline & Overall $(n=119)$ & $\begin{array}{c}\text { Sesame Allergic } \\
(\mathbf{n}=15)\end{array}$ & $\begin{array}{c}\text { Sesame Tolerant } \\
\quad(n=73)\end{array}$ & p-value ${ }^{\dagger}$ \\
\hline Pistachio sensitized ${ }^{\xi}$ & $92(77.3 \%)$ & $15(100 \%)$ & $52(71.2 \%)$ & 0.04 \\
\hline Pistachio allergic ${ }^{t}$ & $9(7.6 \%)$ & $2(13.3 \%)$ & $6(8.2 \%)$ & 0.89 \\
\hline Pistachio $\operatorname{IgE}\left(\mathrm{kU}_{\mathrm{A}} / \mathrm{L}\right)^{*}$ & $11(1.1,40.6)$ & $10.2(2.1,28.9)$ & $7.6(1.0,30.3)$ & 0.76 \\
\hline Hazelnut sensitized ${ }^{\xi}$ & $97(81.5 \%)$ & $15(100 \%)$ & $56(76.7 \%)$ & 0.09 \\
\hline Hazelnut allergic ${ }^{t^{t}}$ & $5(4.2 \%)$ & $2(13.3 \%)$ & $3(4.1 \%)$ & 0.43 \\
\hline Hazelnut $\operatorname{IgE}\left(\mathrm{kU}_{\mathrm{A}} / \mathrm{L}\right)^{*}$ & $10.6(2.7,27.6)$ & $14.4(4.3,22.2)$ & $9.3(2.3,19.5)$ & 0.35 \\
\hline Pecan sensitized ${ }^{\xi}$ & $69(58.0 \%)$ & $11(73.3 \%)$ & $37(50.7 \%)$ & 0.19 \\
\hline Pecan allergic ${ }^{\star}$ & $6(5.0 \%)$ & $1(6.7 \%)$ & $4(5.5 \%)$ & 1.00 \\
\hline Pecan $\operatorname{IgE}\left(\mathrm{kU}_{\mathrm{A}} / \mathrm{L}\right)^{*}$ & $1.7(0.2,9.5)$ & $3.6(0.3,14.4)$ & $1.0(0.2,6.2)$ & 0.37 \\
\hline Walnut sensitized & $91(76.5 \%)$ & $14(93.3 \%)$ & $51(69.9 \%)$ & 0.11 \\
\hline Walnut allergic ${ }^{\star}$ & $8(6.7 \%)$ & $2(13.3 \%)$ & $4(5.5 \%)$ & 0.59 \\
\hline Walnut $\operatorname{IgE}\left(\mathrm{kU}_{\mathrm{A}} / \mathrm{L}\right)^{*}$ & $5.8(0.6,27.0)$ & $8.7(2.6,43.1)$ & $1.9(0.2,21.5)$ & 0.03 \\
\hline
\end{tabular}

Differences in distribution of categorical variables tested via Chi-Squared Test; Differences in distribution of continuous variables tested via Wilcoxon Rank Sum Test

median, $\left(25^{\text {th }}-75^{\text {th }}\right.$ percentile)

$\xi_{\text {Sensitized }}=\operatorname{sIgE} \geq 0.35 \mathrm{kU} / \mathrm{L}$

Allergic = convincing history of symptoms consistent with a hypersensitivity reaction immediately after eating the food

${ }^{\&}$ Fisher's exact test used due to cell counts incompatible with Chi-Squared Test

EGID = eosinophilic gastrointestinal disease

$\mathrm{TN}=$ tree nut 